\title{
Full-term low birth weight infants have differentially hypermethylated DNA related to immune system and organ growth: A comparison with full-term normal birth weight infant
}

Ikuyo Hayashi ( $\square$ ikuyohayashi4119@gmail.com )

https://orcid.org/0000-0003-2238-4028

Ken Yamaguchi

Kyoto University of Graduate School of Medicine

Masahiro Sumitomo

Tajoma KOUNOTORI Perinatal Medical Center

Kenji Takakura

Kokuritsu Byoin Kiko Kyoto Iryo Center

Narumi Nagai

University of Hyogo

Naoki Sakane

Kokuritsu Byoin Kiko Kyoto Iryo Center

\section{Research note}

Keywords: ow birth weight, term infant, gene, DNA methylation, gene ontology (GO), immune system

Posted Date: January 20th, 2020

DOI: https://doi.org/10.21203/rs.2.21228/v1

License: (c) (i) This work is licensed under a Creative Commons Attribution 4.0 International License.

Read Full License

Version of Record: A version of this preprint was published at BMC Research Notes on April 3rd, 2020. See the published version at https://doi.org/10.1186/s13104-020-04961-2. 


\section{Abstract}

Objective: Low birth weight (LBW) is a major public health issue as it results in a higher risk of noncommunicable diseases throughout life. However, the genome-wide DNA methylation patterns of LBW full-term infants (FT-LBWs) are still unclear. The aim of this exploratory study was to compare differences in DNA methylation between FT-LBWs and normal birth weight full-term infants (FT-NBWs) whose mothers were non-smokers and non-complication.

Results: A total of 702 Japanese singleton pregnancies were recruited. The prevalence of preterm LBW and FT-LBW was $3.4 \%$ and $6.1 \%$, respectively. Four FT-LBWs and five FT-NBWs were selected, genomewide DNA methylation analysis including 862,260 methylation CpGs was performed. 483 hyperdifferentially methylated genes (DMGs) and 35 hypo-DMGs were identified in FT-LBW promoter regions. Hyper-DMGs were annotated to 11 biological processes; intriguingly, "macrophage differentiation" (e.g., CASP8 ), "apoptotic mitochondrial changes" (e.g., BH3 ) , "nucleotide-excision repair" (e.g., HUS1) , and "negative regulation of inflammatory response" (e.g., NLRP12 and SHARPIN ) were included within the "immune system" and "DNA metabolism and repair" categories. EREG was classified into "ovarian cumulus expansion" within the "organism growth and organization" category. Our data implies that LBW itself could be associated with epigenetic modulation regarding immune system and cell mature.

\section{Introduction}

Low birth weight (LBW) is widely known to be associated with increased rates of hypertension, diabetes, obesity, stroke, and coronary heart disease in later life [1, 2]. The Developmental Origins Health and Disease $(\mathrm{DOHaD})$ hypothesis suggests that chronic conditions and diseases in later life result from "prenatal programming." In Japan, the prevalence of LBW has reached to approximately $10 \%$ in 2017 , which is the highest level in the developing countries [3]. Therefore, this hypothesis, which explains the effects of in-utero environment in early life, can help us to understand the likely health of the LBW later life. However, to the best of our knowledge, there are no reports of genome-wide methylation studies in LBW full-term infants (FT-LBWs).

Several fetal genome-wide DNA methylation studies have analyzed preterm LBW infants $[4,5,6]$. One such study confirmed by gene enrichment analysis that some biological processes, including neuron differentiation and nervous system development, are associated with gestational age, and reported that gestational length was more highly correlated with DNA methylation than birth weight [5]. Another study of preterm infants attempted to clarify the methylation characteristics of a large cohort [6]. Therefore, previous DNA methylation studies may have captured DNA methylation differences associated with shorter gestation term. Additionally, methylation studies in developed countries have increasingly focused on the effects of higher maternal nutrient intake and greater maternal body mass. There are many reports of specific gene methylation and expression; for example, the expression of IGF2 (insulin-like growth factor 2$)$ is regulated by maternal nutrient intake during fetal development $[7,8,9]$. 
The aim of the present exploratory study was to compare genome-wide DNA methylation between FTLBWs and normal birth weight full-term infants (FT-NBWs) born from non-smoking mothers with no complications during pregnancy. These mother-infant pairs were strictly excluded to control the following conditions reported to affect birth weight and DNA methylation: hypertensive disorder of pregnancy (HDP) [10, 11]; gestational diabetes mellitus (GDM) [12, 13, 14]; maternal infections [15, 16]; tumors [17, 18]; mental disorders $[19,20]$ and smoking $[21,22]$. Ultimately, the maternal backgrounds of FT-LBWs were characterized by lower pre-pregnancy body mass index (BMI). This is the first genome-wide study to investigate DNA methylation patterns in FT-LBWs without the effects of maternal complications and smoking.

\section{Methods}

Study participants

Initial participants in the present study consisted of 702 Japanese women aged $\geq 20$ years who gave birth to a single child between November 2015 and April 2018. Maternal and fetal information was ascertained from medical and prenatal records. The following data was collected: maternal age, previous pregnancies (i.e., primipara or multipara), medical history (e.g., infections, tumors, hypertension, diabetes, and mental disorders), HDP, GDM, smoking, maternal height, pre-pregnancy body weight, gestational weight gain (GWG), fetal gender, gestational age, and birth weight.

The flow chart of this cohort is shown in Fig. 1. Of the 702 subjects, considering the effect on fetal DNA methylation, mothers who delivered at preterm, with complications, and with smoking habit in the preconception period and/or during pregnancy were excluded.

After excluding these mothers, the data of 386 mother-fetal pairs were available for DNA methylation analysis. Overall, FT-LBW (defined as a birth weight $<2,500 \mathrm{~g}$, born at 37-41 weeks of gestation) was 25 subjects. Of these subjects, four FT-LBWs and five FT-NBWs were selected as control by propensity score matching.

Genome-wide DNA methylation analysis

Umbilical cord blood samples were collected at birth and frozen at $-80^{\circ} \mathrm{C}$ until DNA extraction. DNeasy Blood and Tissue Kits (Qiagen) were used to extract DNA from the white cell fraction of cord blood and purified.

For each subject, 865,918 CpG sites across the genome were interrogated using the Illumina Infinium Human Methylation BeadChip (Illumina, USA) [23]. Briefly, 1 g DNA was converted with sodium bisulfite, amplified, fragmented, and hybridized according to the manufacturer's instructions. After implementing bias adjustment by the beta-mixture quantile normalization method, 862,260 CpGs were used for analysis. $\beta$-values were converted into $M$-values ( $\log _{2}$ ratio of $\beta$-value) to account for heteroscedasticity. Differences in methylation in promoter regions relative to UCSC reference sequences were identified 
between FT-LBW and FT-NBW. A Benjamini and Hochberg false discovery rate control was applied. DNA methylation differences were selected based on $\log _{2}$ ratio of $\beta$-value differences $\geq 0.6$.

Gene ontology functional enrichment analysis

We performed functional enrichment analysis using the Database for Annotation, Visualization and Integrated Discovery v6.8 (https://david.ncifcrf.gov/) to identify the biological functions of hyper- and hypomethylated DNA [24]. This approach evaluates DNA methylation data in terms of categories of gene function rather than individual genes. Genes were ranked by magnitude of correlation with a class distinction in the GOTERM_BP algorithm, and an enrichment score was calculated. Significance was defined as $P<0.05$. These biological functions were identified as per the biological functional category they are involved in using the QuickGO browser.

Statistical analysis

Participants' characteristics and baseline data are presented as $n(\%)$ for categorical variables or mean \pm standard deviation for continuous variables. The Chi-square test (categorical data) and the student's ttest (continuous data) in SPSS (Institute, version 23, IBM) was performed to compare characteristics between FT-LBW and FT-NBW groups. Significance was defined as $\mathrm{P}<0.05$.

\section{Results}

The characteristics of participants

Among the total recruited participants, the prevalence of preterm LBW and FT-LBW was $3.4 \%$ and $6.1 \%$, respectively. Table 1 shows the characteristics of participants in the full-term study. Of the total number, 25 were FT-LBW and 361 were FT-NBW. The pre-pregnancy BMI of FT-LBW mothers was significantly lower than that of FT-NBW mothers $(P=0.044)$. Birth weight of infants was also significantly different between FT-LBWs and FT-NBWs $(P<0.001)$. Statistical difference was not found in maternal age, GWG, socioeconomic status, household income or maternal educational level between groups. The gestational age $(P<0.001)$ and infant's gender $(P=0.023)$ were different between FT-LBWs and FT-NBWs. After the adjustment of maternal age and GWG, pre-pregnancy BMI was significantly associated with FT-LBW (adjusted odds ratio: $0.77 ; 95 \%$ confidence interval: $0.63-0.93 ; \mathrm{P}=0.007$ ).

The characteristics of participants for DNA methylation analysis

The pre-pregnancy BMI of FT-LBW mothers was significantly lower than that of FT-NBW mothers (FTLBWs, $17.1 \pm 1.6 \mathrm{~kg} / \mathrm{m}^{2}$ vs. FT-NBWs, $\left.22.2 \pm 2.9 \mathrm{~kg} / \mathrm{m}^{2} ; \mathrm{P}=0.013\right)$. Birth weight was also significantly different between FT-LBWs and FT-NBWs $(2,344 \pm 18 \mathrm{~g}$ vs. 3,011 $\pm 7 \mathrm{~g} ; \mathrm{P}<0.001)$. Less significant difference was found in maternal age, GWG, or gestational age. (Data shown in Table S1)

Identification of DMGs 
A total of 583 hypermethylated reference sequences were determined to correspond to 483 hyperdifferentially methylated genes (DMGs) and 58 hypomethylated reference sequences were determined to correspond to 35 hypo-DMGs in FT-LBW infants, compared to the FT-NBW infants (Figure. 1).

Enrichment analysis and functional annotation

To investigate the effect of birth weight on fetal biological processes, we performed biological enrichment analysis of hyper-DMGs and hypo-DMGs in FT-LBW using the GOTERM_BP algorithm. The 483 hyperDMGs identified were annotated to 11 biological processes in FT-LBW infants. The functions of hypomethylated genes could not be verified. Table 2 shows $\mathrm{GO}$ term and genes of each enrichment biological process. Furthermore, these 11 biological processes could be further categorized as "immune system," "DNA metabolism and repair," and "organism growth and organization".

Of these, five enrichment biological processes were classified into the "immune system" category: clearance of foreign intracellular DNA by conversion of DNA cytidine to uridine (APOBEC3A_B and APOBEC3A), macrophage differentiation (CASP8, CASP10, and BMP4), apoptotic mitochondrial changes (BH3, IFIT2, and AIFM2), negative regulation of viral genome replication (IFI16, ADAR, et al.), and negative regulation of inflammatory response (NLRP12, SHARPIN, et al).

Two enrichment biological processes were identified into the "DNA metabolism and repair" category: purine nucleotide metabolic process (GMPR2, NME/NM23, and GUK1) and nucleotide-excision repair (HUS1, ERCC1, et al).

Another four enrichment biological processes were classified into the "organism growth and organization" category: ovarian cumulus expansion (EREG and BMPR1B), positive regulation of multicellular organism growth (GHR, GHRL, et al), osteoblast differentiation (CREB3L1, FBL, et al), and protein phosphorylation (STRADB, BMPR1B, et al).

\section{Discussion}

This is the first study to compare DNA methylation between FT-LBW and -NBW infants who were born from the mothers without complications and smoking habit. Ultimately, Maternal background of FT-LBWs was characterized by lower pre-pregnancy BMls. It is important to note that participants in this study were select based on strict criteria to exclude maternal factors that may have affected DNA methylation. Our results suggest that LBW itself may be associated with epigenetic modulation of the immune system and cell mature.

We identified the functional categories of hyper-DMGs in FT-LBWs such as macrophage differentiation, apoptotic mitochondrial changes, nucleotide-excision repair, negative regulation of inflammatory response and ovarian cumulus expansion. Interestingly, several genes annotating cell apoptosis; CASP8 and BH3, cell cycle progression; HUS1 and inflammatory response; NLRP12 and SHARPIN were hypermethylated in FT-LBW infants. 
It is well established that CASP8 is an important contributor in the apoptotic pathway. Sequential activation of caspases plays a central role in the execution of cell apoptosis [25]. A previous study was reported that in mice with a deletion in CASP8 in the intestinal epithelium, inflammatory lesions spontaneously developed in the terminal ileum and mice were highly susceptible to colitis [26]. We next observed $\mathrm{BH} 3$ hypermethylation in FT-LBWs. BH3-only proteins have been recognized as essential initiators of apoptosis [27]. Some studies have reported that BH3-only proteins are linked with the promotion of apoptosis in embryonic germ cells, oocytes, follicular granulosa cells, and luteal cells, as well as the regulation of oocyte number and quality in the ovary $[28,29]$.

We also observed increased methylation status of HUS1 in FT-LBW infants. The protein product of this gene forms a complex with RAD9 and RAD1 and functions as a cell cycle checkpoint during replication stress, involved in response to DNA damage [30, 31, 32]. HUS1 DNA hypermethylation could mean that error cells may perpetuate during DNA replication in FT-LBWs. Therefore, LBW could be potentially linked with impaired cell metabolism in their life.

For "negative regulation of inflammatory response" genes, the methylation status of NLRP12 and SHARPIN were increased. NLRP12 downregulates the production of inflammatory cytokines [33]. Some animal study showed that NIrp12-/-mice were highly susceptible to colitis and colitis-associated colon cancer $[34,35]$. SHARPIN plays a role in preventing skin inflammation by inhibiting TNFR1-induced keratinocyte apoptosis [36]. Other study reported that cutaneous inflammation in SHARPIN-deficient mice is autoinflammatory in nature [37]. In data from intrauterine growth restriction (IUGR) animal models which is caused due to maternal malnutrition and/or poor GWG; lower absolute immune organ weights, damaged and jagged villi, decreased villus surface areas, and a smaller number of epithelial goblet cells and lymphocytes were leaded. [38]. Considering together these reports and our results, human LBWs may also have thin and weak intestinal mucosa and high sensitivity that to evoke autoinflammatory.

For the "organism growth and organization" category, the methylation status of EREG, which was annotated to "ovarian cumulus expansion," was increased. The encoded protein may be involved in a wide range of biological processes including inflammation, wound healing, oocyte maturation, and cell proliferation. A previous animal study demonstrated that undernutrition during pregnancy can delay fetal follicular development in sheep [39].

In summary, some alterations in genes annotated to biological processes were identified which could be related to health problems in maternal undernutrition induced LBW infants. We would like to emphasize that it is important to select individual target attributes for preventive intervention even in a small study group and to clarify methylation patterns.

\section{Limitations}

First, the study cohort was small since the criteria for subject selection were specific and stringent. Another limitation is that since this study was a cross-section analysis at birth, it has not been verified whether these methylation differences are actually linked to future health problems. Future observational 
studies are required to clarify the extent to which the DNA methylation status of FT-LBWs persist beyond infancy and are associated with immune development and disease in later life.

\section{Abbreviations}

BMl:body mass index; CpG:cytosine-phosphate-guanine; DMGs:differentially methylated genes; DNA:deoxyribose nucleic acid; DOHaD:The Developmental Origins Health and Disease; FT-:full-term infants; GDM:gestational diabetes mellitus; GO:gene ontology; GWG:gestational weight gain; HDP:hypertensive disorder of pregnancy; LBW:low birth weight; NBW:normal birth weight.

\section{Declarations}

\section{Acknowledgements}

The authors are grateful to all the mothers who took part in this study. The authors also would like to acknowledge all the medical personnel at the Department of Obstetrics and Gynecology and the Division of Preventive Medicine at the National Hospital Organization Kyoto Medical Center for their assistance in data and sample collection.

\section{Authors' contributions}

IH involved all aspect of this study. MS and NN led the design of this study. KY contributed to the analysis and interpretation of the data. NS assisted in the draft of the manuscript. MS, KY, and KT contributed to sample collection. The authors are entirely responsible for the scientific content of the paper. All authors read and approved the final manuscript.

\section{Funding}

This work was supported by the Japan Society for the Promotion of Science (grant number JPI5H0Z90I) and the Smoking Research Foundation, Japan. None of the funding bodies had a role in the study design or analysis.

\section{Ethics approval and consent to participate}

Ethical approval for the study was obtained by the ethics committee of the National Hospital Organization Kyoto Medical Center (approval number: 15-067; approval date: September 20, 2015). All participants provided written informed consent for research. No individual level data is being reported in this manuscript.

\section{Availability of data and materials}

All data generated or analysed during this study are included in this published article and its supplementary information files. 


\section{Consent for publication}

Not applicable.

\section{Competing interests}

The authors declare that they have no competing interests.

\section{References}

1. Barker DJ, Osmond C. Low birth weight and hypertension. BMJ. 1988;297:134- doi: 10.1136/bmj.297.6641.134-b

2. Osmond C, Barker DJ. Fetal, infant, and childhood growth are predictors of coronary heart disease, diabetes, and hypertension in adult men and women. Environmental health perspectives. 2000;108:545-53. doi: 10.1289/ehp.00108s3545.

3. Ministry of Health, Labour and Welfare, Japan: Trends in mean birth weight and percentage of live birth under 2,500g by sex, 1975-2017 (in Japanese). https://www.mhlw.go.jp/toukei/list/dl/811a2.pdf (2019). Accessed 25 Dec 2019.

4. Schroeder JW., Conneely KN, Cubells JF, Kilaru V, Newport DJ, Knight BT, et al. Neonatal DNA methylation patterns associate with gestational age. Epigenetics. 2011;6:1498-504. doi: 10.4161/epi.6.12.18296.

5. Parets SE, Conneely KN, Kilaru V, Fortunato SJ, Syed TA, Saade G, et al. Fetal DNA methylation associates with early spontaneous preterm birth and gestational age. PLoS One. 2013;8:e67489. doi: 10.1371/journal.pone.0067489.

6. Fernando F, Keijser R, Henneman P, van der Kevie AM, Mannens MM, van der Post JA.et al. The idiopathic preterm delivery methylation profile in umbilical cord blood DNA. BMC Genomics. 2015;16:736. doi: 10.1186/s12864-015-1915-4.

7. Feil R, Walter J, Allen ND, Reik W. Developmental control of allelic methylation in the imprinted mouse Igf2 and H19 genes. 1994;120:2933-43.

8. Kovacheva VP, Mellott TJ, Davison JM, Wagner N, Lopez-Coviella I, Schnitzler AC, et al. Gestational choline deficiency causes global and Igf2 gene DNA hypermethylation by up-regulation of Dnmt1 expression. Journal of Biological Chemistry. 2007;282:31777-88. doi: 10.1074/jbc.M705539200.

9. Napoli I, Blusztajn JK, Mellott TJ. Prenatal choline supplementation in rats increases the expression of IGF2 and its receptor IGF2R and enhances IGF2-induced acetylcholine release in hippocampus and frontal cortex. Brain research. 2008;1237:124-35. doi: 10.1016/j.brainres.2008.08.046.

10. Ching T, Ha J, Song MA, Tiirikainen M, Molnar J, Berry MJ, et al. Genome-scale hypomethylation in the cord blood DNAs associated with early onset preeclampsia. Clinical epigenetics. 2015;7:21. doi: 10.1186/s13148-015-0052-x. 
11. Zhu L, Lv R, Kong L, Cheng H, Lan F, Li X. Genome-wide mapping of $5 \mathrm{mC}$ and $5 \mathrm{hmC}$ identified differentially modified genomic regions in late-onset severe preeclampsia: a pilot study. PLoS One. 2015;10:e0134119. doi: 10.1371/journal.pone.0134119.

12. El Hajj N, Pliushch G, Schneider E, Dittrich M, Müller T, Korenkov M, et al. Metabolic programming of MEST DNA methylation by intrauterine exposure to gestational diabetes mellitus. Diabetes. 2013;62:1320-8. doi: 10.2337/db12-0289.

13. Wu P, Farrell WE, Haworth KE, Emes RD, Kitchen MO, Glossop JR, et al. Maternal genome-wide DNA methylation profiling in gestational diabetes shows distinctive disease-associated changes relative to matched healthy pregnancies. Epigenetics. 2018;13:122-8. doi:

10.1080/15592294.2016.1166321.

14. Finer S, Mathews C, Lowe R, Smart M, Hillman S, Foo L, et al. Maternal gestational diabetes is associated with genome-wide DNA methylation variation in placenta and cord blood of exposed offspring. Human molecular genetics. 2015;24:3021-9. doi: 10.1093/hmg/ddv013.

15. Liu Y, Hoyo C, Murphy S, Huang Z, Overcash F, Thompson J, et al. DNA methylation at imprint regulatory regions in preterm birth and infection. American Journal of Obstetrics \& Gynecology. 2013;208:395-e1. doi: 10.1016/j.ajog.2013.02.006.

16. Bierne $H$, Hamon M, Cossart P. Epigenetics and bacterial infections. Cold Spring Harbor perspectives in medicine. 2012;2:a010272. doi: 10.1101/cshperspect.a010272.

17. Baylin SB, Herman JG. DNA hypermethylation in tumorigenesis: epigenetics joins genetics. Trends in genetics. 2000;16:168-74. doi:10.1016/S0168-9525(99)01971-X

18. Esteller M, Herman JG. Cancer as an epigenetic disease: DNA methylation and chromatin alterations in human tumours. The Journal of Pathology. 2002;196:1-7. https://doi.org/10.1002/path.1024

19. Non AL, Binder AM, Kubzansky LD, Michels KB. Genome-wide DNA methylation in neonates exposed to maternal depression, anxiety, or SSRI medication during pregnancy. Epigenetics. 2014;9:964-72. doi: 10.4161/epi.28853.

20. Mansell T, Novakovic B, Meyer B, Rzehak P, Vuillermin P, Ponsonby AL, et al. The effects of maternal anxiety during pregnancy on IGF2/H19 methylation in cord blood. Translational psychiatry. 2016;6:e765. doi: 10.1038/tp.2016.32.

21. Knopik VS, Maccani MA, Francazio S, McGeary JE. The epigenetics of maternal cigarette smoking during pregnancy and effects on child development. Development and psychopathology. 2012;24:1377-90. doi: https://doi.org/10.1017/S0954579412000776.

22. Küpers LK, Xu X, Jankipersadsing SA, Vaez A, La Bastide-van Gemert S, Scholtens S, et al. DNA methylation mediates the effect of maternal smoking during pregnancy on birthweight of the offspring. International journal of epidemiology. 2015;44:1224-37. doi: 10.1093/ije/dyv048

23. Moran S, Arribas C, Esteller M. Validation of a DNA methylation microarray for $850,000 \mathrm{CpG}$ sites of the human genome enriched in enhancer sequences. Epigenomics. 2016;8:389-99. doi: 10.2217/epi.15.114 
24. Sherman BT, Lempicki RA. Systematic and integrative analysis of large gene lists using DAVID bioinformatics resources. Nature protocols. 2009;4:44-57. doi: 10.1038/nprot.2008.211.

25. Inohara N, Koseki T, Hu Y, Chen S, Núñez G. CLARP, a death effector domain-containing protein interacts with caspase-8 and regulates apoptosis. Proceedings of the National Academy of Sciences. 1997;94:10717-22. doi: 10.1073/pnas.94.20.10717.

26. Günther C, Martini E, Wittkopf N, Amann K, Weigmann B, Neumann H, et al. Caspase-8 regulates TNFa-induced epithelial necroptosis and terminal ileitis. Nature. 2011;477:335. doi:

10.1038/nature10400.

27. Huang DC, Strasser A. BH3-only proteins-essential initiators of apoptotic cell death. Cell. 2000;103:839-42. doi: 10.1016/S0092-8674(00)00187-2.

28. Shamas-Din A, Brahmbhatt $H$, Leber B, Andrews DW. BH3-only proteins: Orchestrators of apoptosis. Biochimica et Biophysica Acta (BBA)-Molecular Cell Research. 2011;1813:508-20. https://doi.org/10.1016/j.bbamcr.2010.11.024

29. Hutt KJ. The role of BH3-only proteins in apoptosis within the ovary. Reproduction. 2015;149:R81-9. doi: 10.1530/REP-14-0422.

30. Bai H, Madabushi A, Guan X, Lu AL. Interaction between human mismatch repair recognition proteins and checkpoint sensor Rad9-Rad1-Hus1. DNA repair. 2010;9:478-87. doi:

10.1016/j.dnarep.2010.01.011.

31. Hwang BJ, Jin J, Gunther R, Madabushi A, Shi G, Wilson GM, et al. Association of the Rad9-Rad1Hus1 checkpoint clamp with MYH DNA glycosylase and DNA. DNA repair. 2015;31:80-90. doi: 10.1016/j.dnarep.2015.05.004.

32. Park MJ, Park JH, Hahm SH, Ko SI, Lee YR, Chung JH, et al. Repair activities of human 8-oxoguanine DNA glycosylase are stimulated by the interaction with human checkpoint sensor Rad9-Rad1-Hus1 complex. DNA repair. 2009;8:1190-200. doi: 10.1016/j.dnarep.2009.06.004.

33. Lukens JR, Gurung P, Shaw PJ, Barr MJ, Zaki MH, Brown SA, et al. The NLRP12 sensor negatively regulates autoinflammatory disease by modulating interleukin-4 production in T cells. Immunity. 2015;42:654-64. doi: 10.1016/j.immuni.2015.03.006.

34. Zaki MH, Vogel P, Malireddi RS, Body-Malapel M, Anand PK, Bertin J, et al. The NOD-like receptor NLRP12 attenuates colon inflammation and tumorigenesis. Cancer cell. 2011;20:649-60. doi: 10.1016/j.ccr.2011.10.022.

35. Allen IC, Wilson JE, Schneider M, Lich JD, Roberts RA, Arthur JC, et al. NLRP12 suppresses colon inflammation and tumorigenesis through the negative regulation of noncanonical NF-KB signaling. Immunity. 2012;36:742-54. doi: 10.1016/j.immuni.2012.03.012.

36. Kumari S, Redouane Y, Lopez-Mosqueda J, Shiraishi R, Romanowska M, Lutzmayer S, et al. Sharpin prevents skin inflammation by inhibiting TNFR1-induced keratinocyte apoptosis. Elife. 2014;3:e03422. doi: 10.7554/eLife.03422.001.

37. Potter CS, Wang Z, Silva KA, Kennedy VE, Stearns TM, Burzenski L, et al. Chronic proliferative dermatitis in Sharpin null mice: development of an autoinflammatory disease in the absence of $B$ 
and T lymphocytes and IL4/IL13 signaling. PLoS One. 2014;9:e85666. doi:

10.1371/journal.pone.0085666.

38. Dong L, Zhong X, Ahmad H, Li W, Wang Y, Zhang L, et al. Intrauterine growth restriction impairs small intestinal mucosal immunity in neonatal piglets. Journal of Histochemistry \& Cytochemistry. 2014;62:510-8. doi: 10.1369/0022155414532655.

39. Rae MT, Palassio S, Kyle CE, Brooks AN, Lea RG, Miller DW, et al. Effect of maternal undernutrition during pregnancy on early ovarian development and subsequent follicular development in sheep fetuses. Reproduction. 2001;122:915-22. doi: 10.1530/reprod

\section{Additional Files}

Additional file1: Figure S1. Flow chart illustrating the data of study participants

Additional file2: Table S1. Maternal and infant characteristics in DNA methylation analysis

\section{Tables}

Due to technical limitations, Tables 1-2 are provided in the Supplementary Files section.

\section{Figures}




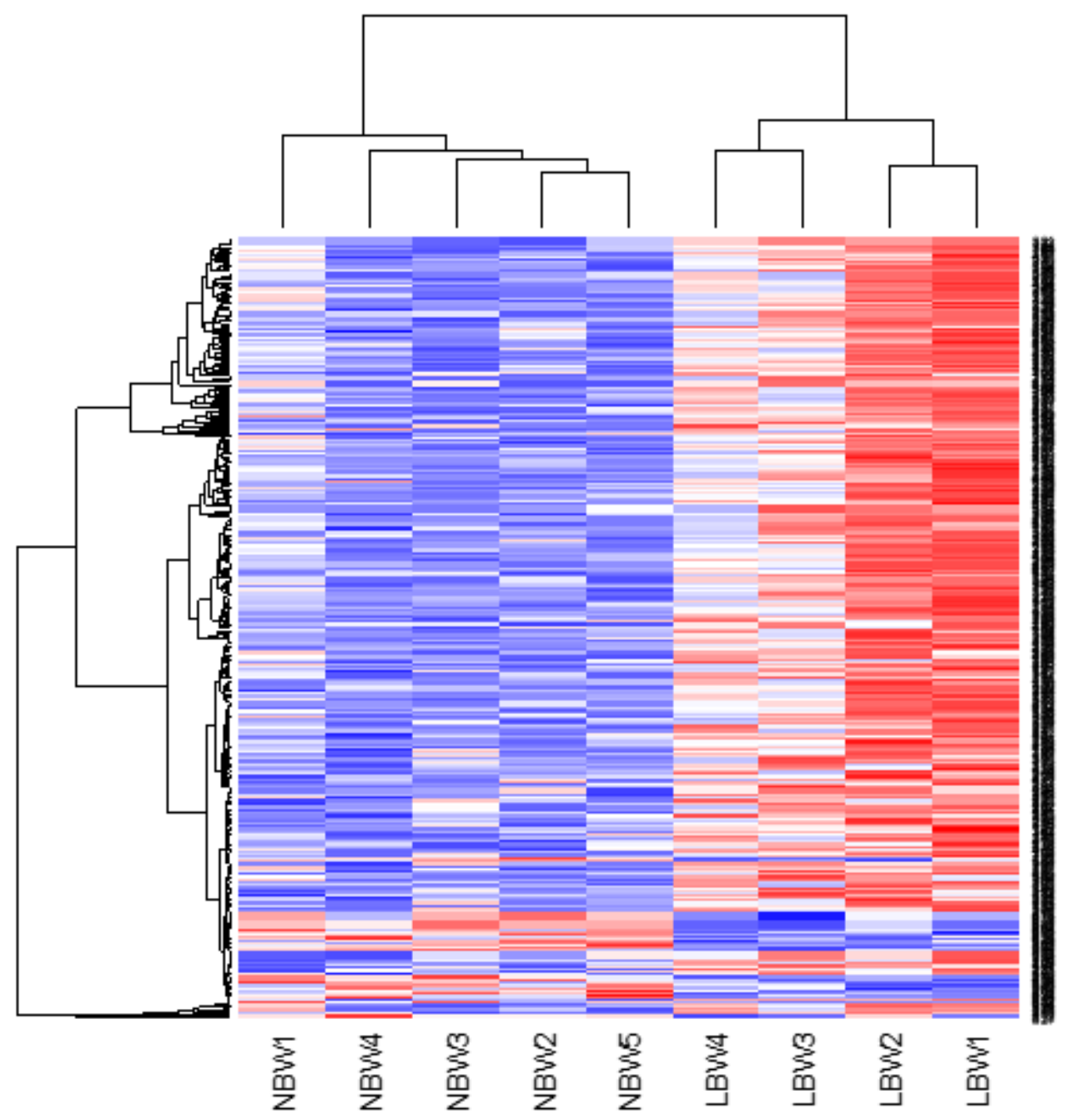

Figure 1

Correlation between DMGs and birth weight. Heat map illustration of the clustering of DMGs with birth weight. Red and blue colors indicate hyper- and hypomethylation, respectively. Average linkage clustering was performed on beta values based on the correlation distance between FT-LBW infants and FT-NBW infants. LBW1-LBW4: full-term low birth weight infants and NBW1-NBW5: full-term normal birth weight infants.

\section{Supplementary Files}

This is a list of supplementary files associated with this preprint. Click to download.

- TableS1BMCResearchNoteslkuyoHayashi.xls

- Table2BMCResearchNoteslkuyoHayashi.xls

- Table1BMCResearchNoteslkuyohayashi.xls 
- FigS1BMCResearchNoteslkuyoHayashi.docx 